\title{
Hyperbolic Function Theory in the Skew-Field of Quaternions
}

\author{
Sirkka-Liisa Eriksson*®a and Heikki Orelma
}

\begin{abstract}
We are studying hyperbolic function theory in the total skewfield of quaternions. Earlier the theory has been studied for quaternion valued functions depending only on three reduced variables. Our functions are depending on all four coordinates of quaternions. We consider functions, called $\alpha$-hyperbolic harmonic, that are harmonic with respect to the Riemannian metric

$$
d s_{\alpha}^{2}=\frac{d x_{0}^{2}+d x_{1}^{2}+d x_{2}^{2}+d x_{3}^{2}}{x_{3}^{\alpha}}
$$

in the upper half space $\mathbb{R}_{+}^{4}=\left\{\left(x_{0}, x_{1}, x_{2}, x_{3}\right) \in \mathbb{R}^{4}: x_{3}>0\right\}$. If $\alpha=2$, the metric is the hyperbolic metric of the Poincaré upper half-space. Hempfling and Leutwiler started to study this case and noticed that the quaternionic power function $x^{m}(m \in \mathbb{Z})$, is a conjugate gradient of a 2-hyperbolic harmonic function. They researched polynomial solutions. Using fundamental $\alpha$-hyperbolic harmonic functions, depending only on the hyperbolic distance and $x_{3}$, we verify a Cauchy type integral formula for conjugate gradient of $\alpha$-hyperbolic harmonic functions. We also compare these results with the properties of paravector valued $\alpha$ hypermonogenic in the Clifford algebra $\mathcal{C} \ell_{0,3}$.
\end{abstract}

Mathematics Subject Classification. Primary 30A05, Secondary 30A45.

Keywords. $\alpha$-hypermonogenic, $\alpha$-hyperbolic harmonic, Laplace-Beltrami operator, Monogenic function, Clifford algebra, Hyperbolic metric, Hyperbolic Laplace operator, Quaternions.

\section{Introduction}

We study quaternion valued twice continuous differentiable functions $f(x)$ defined in an open subset of the full space $\mathbb{R}^{4}$ satisfying the following modified

This article is part of the Topical Collection on FTHD 2018, edited by Sirkka-Liisa Eriksson, Yuri M. Grigoriev, Ville Turunen, Franciscus Sommen and Helmut Malonek.

${ }^{*}$ Corresponding author. 
Cauchy-Riemann system

$$
\begin{gathered}
x_{3}\left(\frac{\partial f_{0}}{\partial x_{0}}-\frac{\partial f_{1}}{\partial x_{1}}-\frac{\partial f_{2}}{\partial x_{2}}-\frac{\partial f_{3}}{\partial x_{3}}\right)+\alpha f_{3}=0, \\
\frac{\partial f_{0}}{\partial x_{m}}=-\frac{\partial f_{m}}{\partial x_{0}} \text { for all } m=1,2,3, \\
\frac{\partial f_{m}}{\partial x_{n}}=\frac{\partial f_{n}}{\partial x_{m}} \text { for all } m, n=1,2,3 .
\end{gathered}
$$

Earlier the theory has been studied for quaternion valued functions depending only on three reduced variables [5]. In case $\alpha=2$, this system was studied by Hempfling and Leutwiler in [11]. Recently, we verified Cauchy type formulas for these function in [6]. In this paper, we study integral formulas and operators produced by these formulas. The results are interesting, since we are building hyperbolic function theory in the full skew field of quaternions. We also develop the theory of paravector valued $\alpha$-hypermonogenic functions in the Clifford algebra $\mathcal{C} \ell_{0,3}$ and find similar integral theorems as in the quaternionic hyperbolic function theory.

\section{Preliminaries}

The skew-field of quaternions $\mathbb{H}$ is four dimensional associative division algebra over reals with an identity $\mathbf{1}$. We denote by $\mathbf{1}, \boldsymbol{i}, \boldsymbol{j}$ and $\boldsymbol{k}$ the generating elements of $\mathbb{H}$ satisfying the relations

$$
\boldsymbol{i}^{2}=\boldsymbol{j}^{2}=\boldsymbol{k}^{2}=\boldsymbol{i} \boldsymbol{j} \boldsymbol{k}=-\mathbf{1} .
$$

The elements $\beta \mathbf{1}$ and $\beta$ are identified for any $\beta \in \mathbb{R}$.

Any quaternion $x$ may be represented with respect to the base $\{\mathbf{1}, \boldsymbol{i}, \boldsymbol{j}, \boldsymbol{k}\}$ by

$$
x=x_{0}+x_{1} \boldsymbol{i}+x_{2} \boldsymbol{j}+x_{3} \boldsymbol{k}
$$

where $x_{0}, x_{1}, x_{2}$ and $x_{3}$ are real numbers. The real vector spaces $\mathbb{R}^{4}$ and $\mathbb{H}$ may be identified.

We denote the upper half space by

$$
\mathbb{R}_{+}^{4}=\left\{\left(x_{0}, x_{1}, x_{2}, x_{3}\right) \mid x_{m} \in \mathbb{R}, m=0,1,2,3 \text { and } x_{3}>0\right\}
$$

and the lower half space by

$$
\mathbb{R}_{-}^{4}=\left\{\left(x_{0}, x_{1}, x_{2}, x_{3}\right) \mid x_{m} \in \mathbb{R}, m=0,1,2,3 \text { and } x_{3}<0\right\} .
$$

We recall that the hyperbolic distance $d_{h}(x, a)$ between the points $x$ and $a$ in $\mathbb{R}_{+}^{4}$ is $d_{h}(x, a)=\operatorname{arcosh}(\lambda(x, a))$ where

$$
\begin{aligned}
\lambda(x, a) & =\frac{\left(x_{0}-a_{0}\right)^{2}+\left(x_{1}-a_{1}\right)^{2}+\left(x_{2}-a_{2}\right)^{2}+x_{3}^{2}+a_{3}^{2}}{2 x_{3} a_{3}} \\
& =\frac{\|x-a\|^{2}+\left\|x-a^{*}\right\|^{2}}{4 x_{3} a_{3}} \\
& =\frac{\|x-a\|^{2}}{2 x_{3} a_{3}}+1=\frac{\left\|x-a^{*}\right\|^{2}}{2 x_{3} a_{3}}-1,
\end{aligned}
$$


and

$$
\begin{aligned}
a^{*} & =\left(a_{0}, a_{1}, a_{2},-a_{3}\right), \\
\|x-a\| & =\sqrt{\left(x_{0}-a_{0}\right)^{2}+\left(x_{1}-a_{1}\right)^{2}+\left(x_{2}-a_{2}\right)^{2}+\left(x_{3}-a_{3}\right)^{2}},
\end{aligned}
$$

(see a proof for example in [12]). Similarly, we may compute the hyperbolic distance between the points $x$ and $a$ in $\mathbb{R}_{-}^{4}$.

The following simple calculation rules

$$
\begin{aligned}
\|x-a\|^{2} & =2 x_{3} a_{3}(\lambda(x, a)-1), \\
\left\|x-a^{*}\right\|^{2} & =2 x_{3} a_{3}(\lambda(x, a)+1), \\
\frac{\|x-a\|^{2}}{\left\|x-a^{*}\right\|^{2}} & =\frac{\lambda(x, a)-1}{\lambda(x, a)+1}=\tanh ^{2}\left(\frac{d_{h}(x, a)}{2}\right),
\end{aligned}
$$

are useful.

We recall that the hyperbolic ball $B_{h}\left(a, r_{h}\right)$ with the hyperbolic center $a$ in $\mathbb{R}_{+}^{4}$ and the radius $r_{h}$ is the same as the Euclidean ball with the Euclidean center

$$
c_{a}\left(r_{h}\right)=\left(a_{0}, a_{1}, a_{2}, a_{3} \cosh r_{h}\right)
$$

and the Euclidean radius $r_{e}=a_{3} \sinh r_{h}$.

The inner product $\langle x, y\rangle$ in $\mathbb{R}^{4}$ is defined as usual by

$$
\langle x, y\rangle=\sum_{m=0}^{3} x_{m} y_{m} .
$$

If $x=x_{0}+x_{1} \boldsymbol{i}+x_{2} \boldsymbol{j}+x_{3} \boldsymbol{k}$ and $y=y_{0}+y_{1} \boldsymbol{i}+y_{2} \boldsymbol{j}+y_{3} \boldsymbol{k}$ are quaternions their inner product is defined similarly as in $\mathbb{R}^{4}$ by

$$
\langle x, y\rangle=\sum_{m=0}^{3} x_{m} y_{m}
$$

The elements

$$
x=x_{0}+x_{1} \boldsymbol{i}+x_{2} \boldsymbol{j}
$$

are called reduced quaternions. The set of reduced quaternions is identified with $\mathbb{R}^{3}$.

The involution ()$^{\prime}$ in $\mathbb{H}$ is the mapping $x \rightarrow x^{\prime}$ defined by

$$
x^{\prime}=x_{0}-x_{1} \boldsymbol{i}-x_{2} \boldsymbol{j}+x_{3} \boldsymbol{k}
$$

and it satisfies

$$
(x y)^{\prime}=x^{\prime} y^{\prime}
$$

for all quaternions $x$ and $y$. The reversion ()$^{*}$ in $\mathbb{H}$ is the mapping $x \rightarrow x^{*}$ defined by

$$
x^{*}=x_{0}+x_{1} \boldsymbol{i}+x_{2} \boldsymbol{j}-x_{3} \boldsymbol{k}
$$

and the conjugation ()$^{-}$in $\mathbb{H}$ is the mapping $x \rightarrow \bar{x}$ defined by $\bar{x}=\left(x^{\prime}\right)^{*}=$ $\left(x^{*}\right)^{\prime}$, that is

$$
\bar{x}=x_{0}-x_{1} \boldsymbol{i}-x_{2} \boldsymbol{j}-x_{3} \boldsymbol{k} .
$$


These involutions satisfy the following product rules

$$
(x y)^{*}=y^{*} x^{*}
$$

and

$$
\overline{x y}=\bar{y} \bar{x}
$$

for all $x, y \in \mathbb{H}$.

The prime involution may be computed as

$$
x^{\prime}=-\boldsymbol{k} x \boldsymbol{k}
$$

for all quaternions $x$. This formula shows, in fact, that the involution ()$^{\prime}$ is the rotation around the $x_{3}$ axes. Similarly, the formulas

$$
\begin{aligned}
\bar{x} & =-\boldsymbol{k} x^{*} \boldsymbol{k}, \\
x^{*} & =-\boldsymbol{k} \bar{x} \boldsymbol{k},
\end{aligned}
$$

hold for all quaternions $x$. Hence we have the identities

$$
x \boldsymbol{k}=\boldsymbol{k} x^{\prime}
$$

and

$$
x^{*} \boldsymbol{k}=\boldsymbol{k} \bar{x}
$$

valid for all quaternions $x$.

The real part of a quaternion $x=x_{0}+x_{1} \boldsymbol{i}+x_{2} \boldsymbol{j}+x_{3} \boldsymbol{k}$ is defined by

$$
\text { Re } x=x_{0}
$$

and the vector part by

$$
\text { Vec } x=x_{1} \boldsymbol{i}+x_{2} \boldsymbol{j}+x_{3} \boldsymbol{k} .
$$

if $\operatorname{Re} x=\operatorname{Re} y=0$, the product rule

$$
x y=-\langle x, y\rangle+x \times y
$$

holds, where $\times$ is the usual cross product.

The mappings $S: \mathbb{H} \rightarrow \mathbb{R}^{3}$ and $T: \mathbb{H} \rightarrow \mathbb{R}$ are defined by

$$
S a=a_{0}+a_{1} \boldsymbol{i}+a_{2} \boldsymbol{j}
$$

and

$$
T a=a_{3}
$$

for $a=a_{0}+a_{1} \boldsymbol{i}+a_{2} \boldsymbol{j}+a_{3} \boldsymbol{k} \in \mathbb{H}$. Using the reversion, we compute the formulas

$$
\begin{aligned}
& S a=\frac{1}{2}\left(a+a^{*}\right)=\frac{1}{2}(a-\boldsymbol{k} \bar{a} \boldsymbol{k}), \\
& T a=-\frac{1}{2}\left(a-a^{*}\right) \boldsymbol{k}=\frac{1}{2}(\boldsymbol{k} \bar{a}-a \boldsymbol{k}) .
\end{aligned}
$$

We use the identities

$$
a b+b a=2 a \operatorname{Re} b+2 b \operatorname{Re} a-2\langle a, b\rangle,
$$




$$
\langle a, b\rangle=\frac{a \bar{b}+b \bar{a}}{2}=\operatorname{Re}(a \bar{b})
$$

and

$$
\frac{1}{2}(a \bar{b} c+c \bar{b} a)=\langle b, c\rangle a-[a, b, c]
$$

valid for all quaternions $a, b$ and $c$. The term $[a, b, c]$, called a triple product, is defined by

$$
[a, b, c]=\langle a, c\rangle b-\langle a, b\rangle c .
$$

If $\operatorname{Re} a=\operatorname{Re} b=\operatorname{Re} c=0$, then (see [10])

$$
[a, b, c]=a \times(b \times c) .
$$

Notice that the triple product is linear with respect to $a, b$ and $c$. Moreover,

$$
\begin{aligned}
{[a, b, c]^{*} } & =\langle a, c\rangle b^{*}-\langle a, b\rangle c^{*} \\
& =\left\langle a^{*}, c^{*}\right\rangle b^{*}-\left\langle a^{*}, b^{*}\right\rangle c^{*} \\
& =\left[a^{*}, b^{*}, c^{*}\right] .
\end{aligned}
$$

\section{Hyperregular Functions}

We define the following hyperbolic generalized Cauchy-Riemann operators $H_{\alpha}^{l}(x)$ and $H_{\alpha}^{r}(x)$ for $x \in \Omega \backslash\left\{x_{3}=0\right\}$ as follows

$$
\begin{array}{ll}
H_{\alpha}^{l} f(x)=D_{l}^{q} f(x)+\alpha \frac{f_{3}}{x_{3}}, & \bar{H}_{\alpha}^{l} f(x)=\bar{D}_{l}^{q} f(x)-\alpha \frac{f_{3}}{x_{3}}, \\
H_{\alpha}^{r} f(x)=D_{r}^{q} f(x)+\alpha \frac{f_{3}}{x_{3}}, & \bar{H}_{\alpha}^{r} f(x)=\bar{D}_{r}^{q} f(x)-\alpha \frac{f_{3}}{x_{3}},
\end{array}
$$

where the parameter $\alpha \in \mathbb{R}$ and

$$
\begin{array}{ll}
D_{l}^{q} f=\frac{\partial f}{\partial x_{0}}+\boldsymbol{i} \frac{\partial f}{\partial x_{1}}+\boldsymbol{j} \frac{\partial f}{\partial x_{2}}+\boldsymbol{k} \frac{\partial f}{\partial x_{3}} & \bar{D}_{l}^{q} f=\frac{\partial f}{\partial x_{0}}-\boldsymbol{i} \frac{\partial f}{\partial x_{1}}-\boldsymbol{j} \frac{\partial f}{\partial x_{2}}-\boldsymbol{k} \frac{\partial f}{\partial x_{3}}, \\
D_{r}^{q} f=\frac{\partial f}{\partial x_{0}}+\frac{\partial f}{\partial x_{1}} \boldsymbol{i}+\frac{\partial f}{\partial x_{2}} \boldsymbol{j}+\frac{\partial f}{\partial x_{3}} \boldsymbol{k}, & \bar{D}_{r}^{q} f=\frac{\partial f}{\partial x_{0}}-\frac{\partial f}{\partial x_{1}} \boldsymbol{i}-\frac{\partial f}{\partial x_{2}} \boldsymbol{j}-\frac{\partial f}{\partial x_{3}} \boldsymbol{k} .
\end{array}
$$

When there is no confusion, we abbreviate $D_{l}^{q} f$ by $D^{q} f$ and $H_{\alpha}^{l}$ by $H_{\alpha}$.

Definition 3.1. Let $\Omega \subset \mathbb{R}^{4}$ be open. A function $f: \Omega \rightarrow \mathbb{H}$ is called $\alpha$ hyperregular, if $f \in \mathcal{C}^{1}(\Omega)$ and

$$
H_{\alpha}^{l} f(x)=H_{\alpha}^{r} f(x)=0
$$

for any $x \in \Omega \backslash\left\{x_{3}=0\right\}$.

We emphasize that a function is $\alpha$-hyperregular provided that it is continuous differentiable in the total open set $\Omega \subset \mathbb{R}^{4}$ and satisfies the preceding equation for all $x$ with $x_{3} \neq 0$.

Computing the components of $H_{\alpha}^{l} f(x)$ and $H_{\alpha}^{r} f(x)$, we obtain 
Proposition 3.2. [6] Let $\Omega \subset \mathbb{R}^{4}$ be open and a function $f: \Omega \rightarrow \mathbb{H}$ continuously differentiable. A function $f$ is $\alpha$-hyperregular in $\Omega$ if and only if

$$
\begin{aligned}
& \frac{\partial f_{0}}{\partial x_{0}}-\frac{\partial f_{1}}{\partial x_{1}}-\frac{\partial f_{2}}{\partial x_{2}}-\frac{\partial f_{3}}{\partial x_{3}}+\alpha \frac{f_{3}}{x_{3}}=0, \quad \text { if } x_{3} \neq 0, \\
& \frac{\partial f_{0}}{\partial x_{m}}=-\frac{\partial f_{m}}{\partial x_{0}} \quad \text { for all } m=1,2,3, \\
& \frac{\partial f_{m}}{\partial x_{n}}=\frac{\partial f_{n}}{\partial x_{m}} \quad \text { for all } m, n=1,2,3 .
\end{aligned}
$$

Our operators are connected to the hyperbolic metric via the hyperbolic Laplace operator as follows.

Proposition 3.3. [6] Let $\Omega \subset \mathbb{R}^{4}$ be open, $x \in \Omega \backslash\left\{x_{3}=0\right\}$ and $f: \Omega \rightarrow \mathbb{R} a$ real twice continuously differentiable function. Then

$$
x_{3}^{\alpha} H_{\alpha}^{l} \bar{H}_{\alpha}^{l} f(x)=x_{3}^{\alpha} H_{\alpha}^{r} \bar{H}_{\alpha}^{r} f(x)=\Delta_{\alpha} f(x)
$$

where the operator

$$
\Delta_{\alpha}=x_{3}^{\alpha}\left(\Delta-\frac{\alpha}{x_{3}} \frac{\partial}{\partial x_{3}}\right)
$$

is the Laplace-Beltrami operator (see [13]) with respect to the Riemannian metric

$$
d s_{\alpha}^{2}=\frac{d x_{0}^{2}+d x_{1}^{2}+d x_{2}^{2}+d x_{3}^{2}}{x_{3}^{\alpha}} .
$$

Definition 3.4. Let $\Omega \subset \mathbb{R}^{4}$ be open. A twice continuously real differentiable function $h: \Omega \rightarrow \mathbb{R}$ is called $\alpha$-hyperbolic harmonic, if

$$
\Delta_{\alpha} h(x)=0
$$

for all $x \in \Omega \backslash\left\{x_{3}=0\right\}$.

We list a couple of simple observations.

Lemma 3.5. Let $\Omega$ be an open subset of $\mathbb{R}^{4}$. If $h: \Omega \rightarrow \mathbb{R}$ is $\alpha$-hyperbolic on $\Omega$ and $h \in \mathcal{C}^{3}(\Omega)$ then the function $\frac{\partial h}{\partial x_{3}}$ satisfies the equation

$$
x_{3}^{2} \Delta h(x)-\alpha x_{3} \frac{\partial h}{\partial x_{3}}(x)+\alpha h(x)=0
$$

for all $x \in \Omega$. Moreover, a twice continuously differentiable function $h: \Omega \rightarrow$ $\mathbb{R}$ satisfies the preceding equation if and only if the function $x_{3}^{-\alpha} h(x)$ is $-\alpha$ hyperbolic harmonic for any $x \in \Omega \backslash\left\{x_{3}=0\right\}$.

Proof. Assume that $x \in \Omega \backslash\left\{x_{3}=0\right\}$. We just compute as follows

$$
\begin{aligned}
\Delta\left(x_{3}^{-\alpha} h\right)+\frac{\alpha}{x_{3}} \frac{\partial h}{\partial x_{3}}= & x_{3}^{-\alpha} \Delta h-\frac{2 \alpha}{x_{3}^{\alpha+1}} \frac{\partial h}{\partial x_{3}}+\alpha(\alpha+1) x_{3}^{-\alpha-2} h \\
& +\frac{\alpha}{x_{3}^{\alpha+1}} \frac{\partial h}{\partial x_{3}}-\alpha^{2} x_{3}^{-\alpha-2} h \\
= & x_{3}^{-\alpha}\left(x_{3}^{2} \Delta h-\alpha x_{3} \frac{\partial h}{\partial x_{3}}+\alpha h\right) .
\end{aligned}
$$


Real valued $\alpha$-hyperbolic functions are especially important, since they produce $\alpha$-hyperregular functions.

Theorem 3.6. [6] Let $\Omega$ be an open subset of $\mathbb{R}^{4}$. If $h$ is $\alpha$-hyperbolic on $\Omega$ then the function $f=\bar{D}^{q} h$ is $\alpha$-hyperregular on $\Omega$. Conversely, if $f$ is $\alpha$ hyperregular on $\Omega$, there exists locally a $\alpha$-hyperbolic function $h$ satisfying $f=\bar{D}^{q} h$.

Theorem 3.7. [6] Let $\Omega$ be an open subset of $\mathbb{R}^{4}$. If a twice continuously differentiable function $f: \Omega \rightarrow \mathbb{H}$ is $\alpha$-hyperregular then the coordinate functions $f_{n}$ for $n=0,1,2$ are $\alpha$-hyperbolic harmonic and $f_{3}$ satisfies the equation

$$
x_{3}^{2} \Delta f_{3}(x)-\alpha x_{3} \frac{\partial f_{3}}{\partial x_{3}}(x)+\alpha(x) f_{3}=0
$$

for any $x \in \Omega$.

The following transformation property is proved in $[1,3]$.

Lemma 3.8. Let $\Omega$ be an open set contained in $\mathbb{R}_{+}^{4}$ or in $\mathbb{R}_{-}^{4}$. A function a twice continuously differentiable function $f: \Omega \rightarrow \mathbb{R}$ is $\alpha$-hyperbolic harmonic if and only if the function $g(x)=x_{3}^{\frac{2-\alpha}{2}} f(x)$ satisfies the equation

$$
\Delta_{2} g+\frac{1}{4}\left(9-(\alpha+1)^{2}\right) g=0 .
$$

\section{Cauchy Type Integral Formulas}

We recall the Stokes theorem for $T$ and $S$-parts proved in [6].

Theorem 4.1. Let $\Omega$ be an open subset of $\mathbb{R}^{4} \backslash\left\{x_{3}=0\right\}$ and $K$ a 3 -chain satisfying $\bar{K} \subset \Omega$. Denote $\left(\nu_{0}, \nu_{1}, \nu_{2}, \nu_{3}\right)$ the outer unit normal and the corresponding quaternion by $\nu=\nu_{0}+\nu_{1} \boldsymbol{i}+\nu_{2} \boldsymbol{j}+\nu_{3} \boldsymbol{k}$. If $f, g \in \mathcal{C}^{1}(\Omega, \mathbb{H})$, then

$$
\int_{\partial K} T(g \nu f+f \nu g) d \sigma=\int_{K} T\left(H_{-\alpha}^{r} g f+g H_{\alpha}^{l} f+H_{\alpha}^{r} f g+f H_{-\alpha}^{l} g\right) d m,
$$

where $d \sigma$ is the surface element and $d m$ the usual Lebesgue volume element in $\mathbb{R}^{4}$.

Theorem 4.2. Let $\Omega$ be an open subset of $\mathbb{R}^{4} \backslash\left\{x_{3}=0\right\}$ and $K$ a 3-chain satisfying $\bar{K} \subset \Omega$. Denote $\left(\nu_{0}, \nu_{1}, \nu_{2}, \nu_{3}\right)$ the outer unit normal and the corresponding quaternion by $\nu=\nu_{0}+\nu_{1} \boldsymbol{i}+\nu_{2} \boldsymbol{j}+\nu_{3} \boldsymbol{k}$. If $f, g \in \mathcal{C}^{1}(\Omega, \mathbb{H})$, then

$$
\int_{\partial K} S(g \nu f+f \nu g) \frac{d \sigma}{x_{3}^{\alpha}}=\int_{K} S\left(H_{\alpha}^{r} g f+g H_{\alpha}^{l} f+H_{\alpha}^{r} f g+f H_{\alpha}^{l} g\right) \frac{d m}{x_{3}^{\alpha}},
$$

where $d \sigma$ is the surface element and $d m$ the usual Lebesgue volume element in $\mathbb{R}^{4}$.

The fundamental $\alpha$-hyperbolic harmonic function, that is the fundamental solution of $\Delta_{\alpha}$, is the following function (see $[4,6,7]$ ). 
Theorem 4.3. Let $x$ and $y$ be points in the upper half space. The fundamental $\alpha$-hyperbolic harmonic function is

$$
E_{\boldsymbol{\alpha}}(x, y)= \begin{cases}\frac{x_{3}^{\frac{\alpha-2}{2}} y_{3}^{\frac{\alpha-2}{2}} Q_{\frac{\alpha}{2}}^{1}(\lambda(x, y))}{2^{\nu+1} \omega_{3}\left(\lambda(x, y)^{2}-1\right)^{\frac{1}{2}}}, & \text { if } \alpha \geq 0, \\ \frac{x_{3}^{\frac{\alpha-2}{2}} y_{3}^{\frac{\alpha-2}{2}} Q_{\frac{-\alpha-2}{2}}^{1}(\lambda(x, y))}{2^{\nu+1} \omega_{3}\left(\lambda(x, y)^{2}-1\right)^{\frac{1}{2}}}, & \text { if } \alpha<0,\end{cases}
$$

where the associated Legendre function is defined by

$$
Q_{\nu}^{1}(\lambda)=\frac{\sqrt{\pi} \Gamma(\nu+2) \lambda^{-\nu}{ }_{2} F_{1}\left(\frac{\nu}{2}, \frac{\nu+1}{2} ; \frac{2 \nu+3}{2} ; \frac{1}{\lambda^{2}}\right)}{2^{\nu+1}\left(\lambda^{2}-1\right)}
$$

and the hypergeometric function by

$$
{ }_{2} F_{1}(a, b ; c ; x)=\frac{1}{\Gamma(c)} \sum_{m=0}^{\infty} \frac{(a)_{m}(b)_{m}}{(c)_{m}} \frac{x^{m}}{m !}
$$

for $|x|<1$.

We remark that the fundamental $\alpha$-hyperbolic harmonic function is unique up to a harmonic function. The reason why we picked the preceding function is that it leads to nice symmetry properties of a kernel, verified after the following theorem.

Theorem 4.4. Denote $r_{h}=d_{h}(x, y), t=\frac{\alpha-2}{2}$ and define

$$
g_{\alpha}\left(r_{h}\right)=\frac{\sqrt{\pi} \Gamma(\nu+2) \cosh ^{-\nu} r_{h}{ }_{2} F_{1}\left(\frac{\nu}{2}, \frac{\nu+1}{2} ; \frac{2 \nu+3}{2} ; \frac{1}{\cosh ^{2} r_{h}}\right)}{2^{\nu+1}},
$$

where

$$
\nu= \begin{cases}\frac{\alpha}{2}, & \text { if } \alpha \geq 0 \\ \frac{-\alpha-2}{2}, & \text { if } \alpha<0 .\end{cases}
$$

The $\alpha$-hyperregular kernel is the function

$$
\begin{aligned}
h_{\alpha}(x, y) & =\bar{D}^{x}\left(E_{\alpha}(x, y)\right) \\
& =x_{3}^{\frac{\alpha-2}{2}} y_{3}^{\frac{\alpha+4}{2}} w_{\alpha}(x, y) s(x, y) \\
& =x_{3}^{\frac{\alpha-2}{2}} y_{3}^{\frac{\alpha+4}{2}} s(x, y) v_{\alpha}(x, y)
\end{aligned}
$$

where

$$
\begin{aligned}
w_{\alpha}(x, y)= & -t \alpha g_{\alpha}\left(r_{h}\right) \boldsymbol{k} \frac{x-S y}{y_{3}} \\
& +\sinh r_{h} g_{\alpha}^{\prime}\left(r_{h}\right)-(t+2) g_{\alpha}\left(r_{h}\right) \cosh r_{h}, \\
v_{\alpha}(x, y)= & -t \alpha g_{\alpha}\left(r_{h}\right) \frac{x-S y}{y_{3}} \boldsymbol{k} \\
& +\sinh r_{h} g_{\alpha}^{\prime}\left(r_{h}\right)-(t+2) g_{\alpha}\left(r_{h}\right) \cosh r_{h},
\end{aligned}
$$

and

$$
s(x, y)=\frac{\left(x-c_{y}\left(r_{h}\right)\right)^{-1}}{x_{3}\left\|x-c_{y}\left(r_{h}\right)\right\|^{2}}
$$


is 2-hyperregular with respect to $x$.

The function $s(x, y)$ is the kernel computed in [2] and in [3].

Clearly, the function $h_{\alpha}(x, y)$ is not symmetrical with respect to $x$ and $y$. However, it has the following symmetry properties.

Proposition 4.5. The function $h_{\alpha}$ has the properties

$$
\begin{gathered}
S\left(h_{\alpha}(y, x)\right)=-S\left(h_{\alpha}(x, y)\right), \\
y_{3}^{\alpha} T h_{-\alpha}(x, y)=-x_{3}^{-\alpha} T h_{\alpha}(y, x),
\end{gathered}
$$

and

$$
y_{3}^{\alpha} T h_{-\alpha}(y, x)=-x_{3}^{-\alpha} T h_{\alpha}(x, y)
$$

for all $x$ and $y$ outside the hyperplane $\left\{\left(u_{0}, u_{1}, u_{3}, u_{3}\right) \in \mathbb{R}^{4} \mid u_{3}=0\right\}$.

Proof. Denote

$$
F_{\alpha}(x, y)=x_{3}^{\frac{\alpha-2}{2}} y_{3}^{\frac{\alpha-2}{2}} G_{\alpha}(\lambda(x, y)) .
$$

If $m=0,1,2$, then

$$
\begin{aligned}
\frac{\partial F_{\alpha}(x, y)}{\partial x_{m}} & =x_{3}^{\frac{\alpha-2}{2}} y_{3}^{\frac{\alpha-2}{2}} G_{\alpha}^{\prime}(\lambda((x, y))) \frac{\partial \lambda(x, y)}{\partial x_{m}} \\
& =x_{3}^{\frac{\alpha-2}{2}} y_{3}^{\frac{\alpha-2}{2}} G_{\alpha}^{\prime}(\lambda(x, y)) \frac{x_{m}-y_{m}}{x_{3} y_{3}} \\
& =-x_{3}^{\frac{\alpha-2}{2}} y_{3}^{\frac{\alpha-2}{2}} G_{\alpha}^{\prime}(\lambda(y, x)) \frac{y_{m}-x_{m}}{x_{3} y_{3}} \\
& =-\frac{\partial F_{\alpha}(y, x)}{\partial y_{m}}
\end{aligned}
$$

The last properties follow from the tedious calculations

$$
\begin{aligned}
& y_{3}^{\alpha} \partial_{x_{3}} F_{-\alpha}(x, y)+x_{3}^{-\alpha} \partial_{y_{3}} F_{\alpha}(x, y)=0, \\
& y_{3}^{\alpha} \partial_{y_{3}} F_{-\alpha}(x, y)+x_{3}^{-\alpha} \partial_{x_{3}} F_{\alpha}(x, y)=0
\end{aligned}
$$

which are done in [7].

We recall the integral formulas for $S$ - and $T$-parts verified in [6].

Theorem 4.6. Let $\Omega$ and be an open subsets of $\mathbb{R}_{+}^{4}\left(\right.$ or $\left.\mathbb{R}_{-}^{4}\right)$. Assume that $K$ is an open subset of $\Omega$ and $\bar{K} \subset \Omega$ is a compact set with the smooth boundary. Let $\left(\nu_{0}, \nu_{1}, \nu_{2}, \nu_{3}\right)$ be the outer unit normal and denote the corresponding quaternion by $\nu=\nu_{0}+\nu_{1} \boldsymbol{i}+\nu_{2} \boldsymbol{j}+\nu_{3} \boldsymbol{k}$. If $f$ is $\alpha$-hyperregular in $\Omega$ and $a \in K$, then

$$
\begin{aligned}
S f(a) & =-\frac{1}{2} \int_{\partial K} S\left(h_{\alpha}(x, a) \nu f+f \nu h_{\alpha}(x, a)\right) \frac{d \sigma}{x_{3}^{\alpha}} \\
& =\int_{\partial K} S\left[h_{\alpha}(x, a), \bar{\nu}, f\right] \frac{d \sigma}{x_{3}^{\alpha}}-\int_{\partial K} S h_{\alpha}(x, a)\langle\bar{\nu}, f\rangle d \sigma
\end{aligned}
$$

and 


$$
\begin{aligned}
T f(a) & =-\frac{a_{3}^{\alpha}}{2} \int_{\partial K} T\left(h_{-\alpha}(x, a) \nu f+f \nu h_{-\alpha}(x, a)\right) d \sigma \\
& =a_{3}^{\alpha}\left(\int_{\partial K} T\left[h_{-\alpha}(x, a), \bar{\nu}, f\right] d \sigma-\int_{\partial K} T h_{-\alpha}(x, a)\langle\bar{\nu}, f\rangle d \sigma\right) .
\end{aligned}
$$

If we combine these formulas we obtain a new formula.

Theorem 4.7. Let $\Omega$ and be an open subsets of $\mathbb{R}_{+}^{4}$ (or $\left.\mathbb{R}_{-}^{4}\right)$. Assume that $K$ is an open subset of $\Omega$ and $\bar{K} \subset \Omega$ is a compact set with the smooth boundary. Let $\left(\nu_{0}, \nu_{1}, \nu_{2}, \nu_{3}\right)$ be the outer unit normal and denote the corresponding quaternion by $\nu=\nu_{0}+\nu_{1} \boldsymbol{i}+\nu_{2} \boldsymbol{j}+\nu_{3} \boldsymbol{k}$. If $f$ is $k$-hyperregular in $\Omega$ and $a \in K$, then

$$
f(a)=\int_{\partial K} R(x, a, \nu, f) d \sigma+\int_{\partial K} h_{k}(a, x)\langle\bar{\nu}, f\rangle \frac{d \sigma}{x_{3}^{\alpha}}
$$

where

$$
\begin{aligned}
R(x, a, \nu, S f)= & -\left\langle x_{3}^{-\alpha} S h_{\alpha}(a, x), S f\right\rangle S \bar{\nu}+\left\langle a_{3}^{\alpha} S h_{-\alpha}(a, x), S f\right\rangle T \nu \boldsymbol{k} \\
& +\left\langle x_{3}^{-\alpha} S h_{\alpha}(a, x), S \bar{\nu}\right\rangle S f-a_{3}^{\alpha} T h_{-\alpha}^{a}(a, x) T \nu S f
\end{aligned}
$$

and

$$
T(R(x, a, \nu, T f \boldsymbol{k}))=\left\langle a_{3}^{\alpha} S h_{-\alpha}(a, x), S f\right\rangle T \nu+\left\langle a_{3}^{\alpha} S h_{-\alpha}(a, x), S \bar{\nu}\right\rangle T f .
$$

Proof. We combine the preceding integral formulas using the formula

$$
f(a)=S f(a)+T f(a) \boldsymbol{k} .
$$

We introduce the following notation

$$
\begin{aligned}
B & =-\int_{\partial K} x_{3}^{-\alpha} S h_{\alpha}(x, a)\langle\bar{\nu}, f\rangle d \sigma-\int_{\partial K} a_{3}^{\alpha} T h_{-\alpha}(x, a) \boldsymbol{k}\langle\bar{\nu}, f\rangle d \sigma \\
& =-\int_{\partial K}\left(x_{3}^{-\alpha} S h_{\alpha}(x, a)+a_{3}^{\alpha} T h_{-\alpha}(x, a) \boldsymbol{k}\right)\langle\bar{\nu}, f\rangle d \sigma .
\end{aligned}
$$

Applying the symmetry properties of the kernels we deduce

$$
\begin{aligned}
B & =\int_{\partial K}\left(x_{3}^{-\alpha} S h_{\alpha}(a, x)+x_{3}^{-\alpha} T h_{\alpha}(a, x) \boldsymbol{k}\right)\langle\bar{\nu}, f\rangle d \sigma \\
& =\int_{\partial K} x_{3}^{-\alpha} h_{\alpha}(a, x)\langle\bar{\nu}, f\rangle d \sigma .
\end{aligned}
$$

Applying the properties (2.4) and (2.5), we obtain

$$
\begin{aligned}
R(x, a, \nu, f)= & S\left(\left[x_{3}^{-\alpha} h_{\alpha}(x, a), \bar{\nu}, f\right]\right)+T\left(\left[a_{3}^{\alpha} h_{-\alpha}(x, a), \bar{\nu}, f\right]\right) \boldsymbol{k} \\
= & \frac{1}{2}\left(\left[x_{3}^{-\alpha} h_{\alpha}(x, a), \bar{\nu}, f\right]+\left[x_{3}^{-\alpha} h_{\alpha}(x, a), \bar{\nu}, f\right]^{*}\right) \\
& +\frac{1}{2}\left(\left[a_{3}^{\alpha} h_{-\alpha}(x, a), \bar{\nu}, f\right]-\left[a_{3}^{\alpha} h_{-\alpha}(x, a), \bar{\nu}, f\right]^{*}\right) \\
= & \frac{1}{2}\left[x_{3}^{-\alpha} h_{\alpha}(x, a)+a_{3}^{\alpha} h_{-\alpha}(x, a), \bar{\nu}, f\right] \\
& +\frac{1}{2}\left[x_{3}^{-\alpha} h_{\alpha}(x, a)-a_{3}^{\alpha} h_{-\alpha}(x, a), \bar{\nu}, f\right]^{*} .
\end{aligned}
$$


Hence

$$
R(x, a, \nu, f)=R(x, a, \nu, S f)+R(x, a, \nu, T f \boldsymbol{k}) .
$$

Using the definition of the triple product we infer

$$
\begin{aligned}
R(x, a, \nu, S f)= & \frac{1}{2}\left\langle x_{3}^{-\alpha} h_{\alpha}(x, a)+a_{3}^{\alpha} h_{-\alpha}(x, a), S f\right\rangle \bar{\nu} \\
& -\frac{1}{2}\left\langle x_{3}^{-\alpha} h_{\alpha}(x, a)+a_{3}^{\alpha} h_{-\alpha}(x, a), \bar{\nu}\right\rangle S f \\
& +\frac{1}{2}\left\langle x_{3}^{-\alpha} h_{\alpha}(x, a)-a_{3}^{\alpha} h_{-\alpha}(x, a), S f\right\rangle \nu^{\prime} \\
& -\frac{1}{2}\left\langle x_{3}^{-\alpha} h_{\alpha}(x, a)-a_{3}^{\alpha} h_{-\alpha}(x, a), \bar{\nu}\right\rangle S f \\
= & \left\langle x_{3}^{-\alpha} h_{\alpha}(x, a), S f\right\rangle S \bar{\nu}-\left\langle a_{3}^{\alpha} h_{-\alpha}(x, a), S f\right\rangle T \nu \boldsymbol{k} \\
& -\left\langle x_{3}^{-\alpha} h_{\alpha}(x, a), \bar{\nu}\right\rangle S f \\
= & \left\langle x_{3}^{-\alpha} S h_{\alpha}(x, a), S f\right\rangle S \bar{\nu}-\left\langle a_{3}^{\alpha} S h_{-\alpha}(x, a), S f\right\rangle T \nu \boldsymbol{k} \\
& -\left\langle x_{3}^{-\alpha} S h_{\alpha}(x, a), S \bar{\nu}\right\rangle S f+x_{3}^{\alpha} T h_{-\alpha}(x, a) T \nu S f .
\end{aligned}
$$

Using the symmetry properties, we obtain

$$
\begin{aligned}
R(x, a, \nu, S f)= & -\left\langle x_{3}^{-\alpha} S h_{\alpha}(a, x), S f\right\rangle S \bar{\nu}+\left\langle a_{3}^{\alpha} S h_{-\alpha}(a, x), S f\right\rangle T \nu \boldsymbol{k} \\
& +\left\langle x_{3}^{-\alpha} S h_{\alpha}(a, x), S \bar{\nu}\right\rangle S f-a_{3}^{\alpha} T h_{-\alpha}^{a}(a, x) T \nu S f .
\end{aligned}
$$

In order to shorten the notations, we abbreviate $g=T f \boldsymbol{k}$. Then we simply compute

$$
\begin{aligned}
R(x, a, \nu, g)= & \frac{1}{2}\left\langle x_{3}^{-\alpha} h_{\alpha}(x, a)+a_{3}^{\alpha} h_{-\alpha}(x, a), g\right\rangle \bar{\nu} \\
& -\frac{1}{2}\left\langle x_{3}^{-\alpha} h_{\alpha}(x, a)+a_{3}^{\alpha} h_{-\alpha}(x, a), \bar{\nu}\right\rangle g \\
& +\frac{1}{2}\left\langle x_{3}^{-\alpha} h_{\alpha}(x, a)-a_{3}^{\alpha} h_{-\alpha}(x, a), g\right\rangle \nu^{\prime} \\
& +\frac{1}{2}\left\langle x_{3}^{-\alpha} h_{\alpha}(x, a)-a_{3}^{\alpha} h_{-\alpha}(x, a), \bar{\nu}\right\rangle g \\
= & \left\langle x_{3}^{-\alpha} h_{\alpha}(x, a), g\right\rangle S \bar{\nu}-\left\langle a_{3}^{\alpha} h_{-\alpha}(x, a), g\right\rangle T \nu \boldsymbol{k} \\
& -\left\langle a_{3}^{\alpha} h_{-\alpha}(x, a), \bar{\nu}\right\rangle g \\
= & \left\langle x_{3}^{-\alpha} T h_{\alpha}(x, a) \boldsymbol{k}, g\right\rangle S \bar{\nu}-\left\langle a_{3}^{\alpha} T h_{-\alpha}(x, a) \boldsymbol{k}, g\right\rangle T \nu \boldsymbol{k} \\
& -\left\langle a_{3}^{\alpha} h_{-\alpha}(x, a), \bar{\nu}\right\rangle g .
\end{aligned}
$$

Symmetry properties imply that

$$
R(x, a, \nu, g)=\left\langle a_{3}^{\alpha} S h_{-\alpha}(a, x), S \bar{\nu}\right\rangle g-a_{3}^{\alpha} T h_{-\alpha}(a, x) T f S \bar{\nu} .
$$

Corollary 4.8. Let $\Omega$ be an open subsets of $\mathbb{R}_{+}^{4}$ (or $\left.\mathbb{R}_{-}^{4}\right)$. Assume that $K$ is an open subset of $\Omega$ and $\bar{K} \subset \Omega$ is a compact set with the smooth boundary. Let $\left(\nu_{0}, \nu_{1}, \nu_{2}, \nu_{3}\right)$ be the outer unit normal and denote the corresponding 
quaternion by $\nu=\nu_{0}+\nu_{1} \boldsymbol{i}+\nu_{2} \boldsymbol{j}+\nu_{3} \boldsymbol{k}$. If $f$ is $k$-hyperregular in $\Omega$ and $a \in K$, then the functions

$$
r_{1}(a)=\int_{\partial K} R(x, a, \nu, f) d \sigma
$$

and

$$
r_{2}(a)=\int_{\partial K} h_{k}(a, x)\langle\bar{\nu}, f\rangle \frac{d \sigma}{x_{3}^{\alpha}}
$$

are $\alpha$-hyperregular and $f=r_{1}+r_{2}$.

Theorem 4.9. Let $\Omega$ be an open subsets of $\mathbb{R}_{+}^{4}\left(\right.$ or $\left.\mathbb{R}_{-}^{4}\right)$. Assume that $K$ is an open subset of $\Omega$ and $\bar{K} \subset \Omega$ is a compact set with the smooth boundary. Let $\left(\nu_{0}, \nu_{1}, \nu_{2}, \nu_{3}\right)$ be the outer unit normal and denote the corresponding quaternion by $\nu=\nu_{0}+\nu_{1} \boldsymbol{i}+\nu_{2} \boldsymbol{j}+\nu_{3} \boldsymbol{k}$. If $f: \partial K \rightarrow \mathbb{H}$ is a continuous function then the function

$$
r_{2}(a)=\int_{\partial K} h_{\alpha}(a, x)\langle\bar{\nu}, f\rangle \frac{d \sigma}{x_{3}^{\alpha}}
$$

is $\alpha$-hyperregular for all $a \in K$.

Theorem 4.10. Let $\Omega$ be an open subsets of $\mathbb{R}_{+}^{4}$ (or $\left.\mathbb{R}_{-}^{4}\right)$. Assume that $K$ is an open subset of $\Omega$ and $\bar{K} \subset \Omega$ is a compact set with the smooth boundary. Let $\left(\nu_{0}, \nu_{1}, \nu_{2}, \nu_{3}\right)$ be the outer unit normal and denote the corresponding quaternion by $\nu=\nu_{0}+\nu_{1} \boldsymbol{i}+\nu_{2} \boldsymbol{j}+\nu_{3} \boldsymbol{k}$. If $f: \partial K \rightarrow \mathbb{H}$ is a continuous function, then the function

$$
S r_{1}(a)=\int_{\partial K} S(R(x, a, \nu, f)) d \sigma
$$

is $k$-hyperbolic harmonic for all $a \in K$ and

$$
\operatorname{Tr}_{1}(a)=\int_{\partial K} T(R(x, a, \nu, f)) d \sigma
$$

satisfies the equation

$$
x_{3}^{2} \Delta h-\alpha x_{3} \frac{\partial h}{\partial x_{3}}+\alpha h=0
$$

and $a_{3}^{-\alpha} \operatorname{Tr}_{1}$ is $-\alpha$-hyperbolic harmonic.

We consider the Teodorescu and Cauchy type operators in subsequent papers. Also the case $a \in \mathbb{R}_{+}^{4} \backslash K$ involves some technical assumptions and left for later work.

\section{Comparison of $\alpha$-Hyperregular and $\alpha$-Hypermonogenic Functions}

The universal real Clifford algebra $\mathcal{C} \ell_{0,3}$ is a real associated algebra with a unit 1 and is generated by $e_{1}, e_{2}$ and $e_{3}$ satisfying the relation

$$
e_{s} e_{t}+e_{t} e_{s}=-2 \delta_{s t} \mathbf{1}
$$


where $\delta_{s t}$ is the usual Kronecker delta and $s, t=1,2,3$. We denote $r \mathbf{1}$ briefly by $r \in \mathbb{R}$.

The elements

$$
x=x_{0}+x_{1} e_{1}+x_{2} e_{2}+x_{3} e_{3}
$$

for $x_{0}, x_{1}, x_{2}, x_{3} \in \mathbb{R}$ are called paravectors. The real number $x_{0}$ is the real part of the paravector $x$.

The main involution in $\mathcal{C} \ell_{0,3}$ is the mapping $a \rightarrow a^{\prime}$ defined by $e_{s}^{\prime}=-e_{s}$ for $s=1, \ldots, 3$ and extended to the total algebra by linearity and the product rule $(a b)^{\prime}=a^{\prime} b^{\prime}$. Similarly the reversion is the mapping $a \rightarrow a^{*}$ defined by $e_{s}^{*}=-e_{s}$ for $s=1, \ldots, 3$ and extended to the total algebra by linearity and the product rule $(a b)^{*}=b^{*} a^{*}$. The conjugation is the mapping $a \rightarrow \bar{a}$ defined by $\bar{a}=\left(a^{\prime}\right)^{*}=\left(a^{*}\right)^{\prime}$.

Any element $w$ in $\mathcal{C} \ell_{0,3}$ may be written as

$$
w=w_{0}+w_{1} e_{1}+w_{2} e_{2}+w_{3} e_{3}+w_{12} e_{12}+w_{13} e_{13}+w_{23} e_{23}+w_{123} e_{123},
$$

where $e_{m n}=e_{m} e_{n}$ for $1 \leq m<n \leq 3$ and $e_{123}=e_{1} e_{2} e_{3}$. The element $e_{123}$, denoted by $I$, is commuting with all elements and $\left(e_{1} e_{2} e_{3}\right)^{2}=1$.

We recall that $C \ell_{0,1}$ may be identified with the field of complex numbers. The universal Clifford algebra $\mathcal{C} \ell_{0,2}$ may be identified with the quaternions, by setting $\boldsymbol{i}=e_{1}, \boldsymbol{j}=e_{2}$ and $\boldsymbol{k}=e_{1} e_{2}$. This identification we used in the first section when we defined involutions.

We generalize the imaginary part of a complex number to $\mathcal{C} \ell_{0,3}$ by decomposing any element $a \in \mathcal{C} \ell_{0,3}$ as

$$
a=b+c e_{3}
$$

for $b, c \in \mathcal{C} \ell_{0,2}$. The mappings $P: \mathcal{C} \ell_{0,3} \rightarrow \mathcal{C} \ell_{0,2}$ and $Q: \mathcal{C} \ell_{0,3} \rightarrow \mathcal{C} \ell_{0,2}$ are defined in [9] by

$$
P a=b, \quad Q a=c .
$$

In order to compute the $P$ - and $Q$ - parts we use the involution $a \rightarrow \widehat{a}$ defined by $\widehat{e}_{i}=(-1)^{\delta_{s 3}} e_{i}$ for $s=1,2,3$ and extended to the total algebra by linearity and the product rule $\widehat{a b}=\widehat{a} \widehat{b}$. Then we obtain the formulas

$$
P a=\frac{1}{2}(a+\widehat{a})
$$

and

$$
Q a=-\frac{1}{2}(a-\widehat{a}) e_{3} .
$$

The following calculation rules [9] hold

$$
\begin{aligned}
P(a b) & =(P a) P b+(Q a) Q\left(b^{\prime}\right) \\
Q(a b) & =(P a) Q b+(Q a) P^{\prime}(b) \\
& =a Q b+(Q a) b^{\prime} .
\end{aligned}
$$

Note that if $a \in \mathcal{C} \ell_{0,3}$, then

$$
a^{\prime} e_{3}=e_{3} \widehat{a}
$$


Moreover if $a \in \mathcal{C} \ell_{0,2}$ then

$$
a e_{3}=e_{3} a^{\prime}
$$

We consider functions $f: \Omega \rightarrow \mathcal{C} \ell_{0,3}$, defined on an open subset $\Omega$ of $\mathbb{R}^{4}$, and assume that its components are continuously differentiable. The left Dirac operator (also called the Cauchy-Riemann operator) in $\mathcal{C} \ell_{0,3}$ is defined by

$$
D_{l} f=\sum_{s=0}^{3} e_{s} \frac{\partial f}{\partial x_{s}}
$$

and the right Dirac operator by

$$
D_{r} f=\sum_{s=0}^{3} \frac{\partial f}{\partial x_{s}} e_{s} .
$$

Their conjugate operators $\overline{D_{l}}$ and $\overline{D_{r}}$ are defined by

$$
\overline{D_{l}} f=\sum_{s=0}^{3} \overline{e_{s}} \frac{\partial f}{\partial x_{s}}, \quad \overline{D_{r}} f=\sum_{s=0}^{3} \frac{\partial f}{\partial x_{s}} \overline{e_{s}} .
$$

The modified Dirac operators $M_{\alpha}^{l}, \bar{M}_{\alpha}^{l}, M_{\alpha}^{r}$ and $\bar{M}_{\alpha}^{r}$, introduced in $[8,9]$, are defined in $\left\{\left(x_{0}, x_{1}, x_{2}, x_{3}\right) \in \Omega \mid x_{3} \neq 0\right\}$ by

$$
\begin{aligned}
& M_{\alpha}^{l} f(x)=D_{l} f(x)+\alpha \frac{Q^{\prime} f}{x_{3}}, \bar{M}_{\alpha}^{l} f(x)=\overline{D_{l}} f(x)-\alpha \frac{Q^{\prime} f}{x_{3}}, \\
& M_{\alpha}^{r} f(x)=D_{r} f(x)+\alpha \frac{Q f}{x_{3}},{\overline{M_{\alpha}}}^{r} f(x)=\overline{D_{r}} f(x)+\alpha \frac{Q f}{x_{3}},
\end{aligned}
$$

where $(Q f)^{\prime}=Q^{\prime} f$. The operator $M_{2}^{l}$ is also abbreviated by $M$.

Definition 5.1. Let $\Omega \subset \mathbb{R}^{4}$ be open. A function $f: \Omega \rightarrow C \ell_{0,3}$ is called left $\alpha$-hypermonogenic if $f \in \mathcal{C}^{1}(\Omega)$ and

$$
M_{\alpha}^{l} f(x)=0
$$

for any $x \in\left\{x \in \Omega \mid x_{3} \neq 0\right\}$. The right $\alpha$-hypermonogenic functions are defined similarly. The 2-left hypermonogenic functions are called hypermonogenic functions. A twice continuously differentiable function $f: \Omega \rightarrow \mathcal{C} \ell_{0,3}$ is called $\alpha$-hyperbolic harmonic if $\bar{M}_{\alpha}^{l} M_{\alpha}^{l} f=0$.

Computing the components of $M_{\alpha}^{l} f(x)$ and $M_{\alpha}^{r} f(x)$, we obtain

Theorem 5.2. Let $\Omega \subset \mathbb{R}^{4}$ be open and a function $f: \Omega \rightarrow \mathcal{C} \ell_{0,3}$ continuously differentiable. If $f$ is paravector valued then $f$ is $\alpha$-hypermonogenic in $\Omega$ if and only if

$$
\begin{aligned}
& \frac{\partial f_{0}}{\partial x_{0}}-\frac{\partial f_{1}}{\partial x_{1}}-\frac{\partial f_{2}}{\partial x_{2}}-\frac{\partial f_{3}}{\partial x_{3}}+\alpha \frac{f_{3}}{x_{3}}=0, \quad \text { if } x_{3} \neq 0, \\
& \frac{\partial f_{0}}{\partial x_{m}}=-\frac{\partial f_{m}}{\partial x_{0}} \quad \text { for all } m=1,2,3, \\
& \frac{\partial f_{m}}{\partial x_{n}}=\frac{\partial f_{n}}{\partial x_{m}} \quad \text { for all } m, n=1,2,3 .
\end{aligned}
$$

Applying Proposition 3.1 we obtain the result. 
Theorem 5.3. Let $\Omega \subset \mathbb{R}^{4}$ be open and a function $f=\left(f_{0}, f_{1}, f_{2}, f_{3}\right): \Omega \rightarrow$ $\mathbb{R}^{4}$ continuously differentiable. Then the function $f_{0}+f_{1} \boldsymbol{i}+f_{2} \boldsymbol{j}+f_{3} \boldsymbol{k}$ is $\alpha$ hyperregular in $\Omega$ if and only if the $f_{0}+f_{1} e_{1}+f_{2} e_{2}+f_{3} e_{3}$ is $\alpha$-hypermonogenic in $\Omega$.

We recall the Cauchy type formula for $\alpha$-hypermonogenic functions.

Theorem 5.4. [7] Let $\Omega$ be an open subset of $\mathbb{R}_{+}^{4}$ and $K \subset \Omega$ be a smoothly bounded compact set. Denote $\left(\nu_{0}, \nu_{1}, \nu_{2}, \nu_{3}\right)$ the outer unit normal and the corresponding paravector by $\nu=\nu_{0}+\nu_{1} e_{1}+\nu_{2} e_{2}+\nu_{3} e_{3}$. If $f$ is $\alpha$-hypermonogenic in $\Omega$ and $a \in K$, then

$$
f(a)=\int_{\partial K}\left(x_{3}^{-\alpha} h_{\alpha}^{a}(a, x) P(\nu f)+a_{3}^{\alpha} h_{-\alpha}^{a}(x, a) e_{3} Q^{\prime}(\nu f)\right) d \sigma
$$

where

$$
h_{\alpha}(a, x)=\bar{D}^{a} E_{\alpha}(a, x)
$$

and $h_{\alpha}(a, x)$ and $a_{3}^{\alpha} h_{-\alpha}(a, x) e_{3}$ are the $\alpha$-hypermonogenic kernels with respect to $a$.

Using this formula we may verify the formula also for paravector valued functions. Before this, we present three preliminary results.

Lemma 5.5. Let $a \in \Omega \rightarrow \mathcal{C} \ell_{0,3}$. Then

$$
\begin{aligned}
& P\left(a^{*}\right)=(P(a))^{*}, \\
& Q\left(a^{*}\right)=\overline{Q(a)}
\end{aligned}
$$

and

$$
\left(Q^{\prime}\left(a^{*}\right)\right)^{*}=Q(a) .
$$

Proof. Assume that $a \in \mathcal{C} \ell_{0,3}$. Since

$$
a=P a+Q a e_{3}
$$

and $e_{3} Q a=Q^{\prime} a e_{3}$ then

$$
\begin{aligned}
a^{*} & =(P a)^{*}+e_{3}(Q a)^{*} \\
& =(P a)^{*}+\left((Q a)^{*}\right)^{\prime} e_{3} .
\end{aligned}
$$

Noticing that $\left((Q a)^{*}\right)^{\prime}=\overline{Q a}$ we conclude

$$
P\left(a^{*}\right)=(P a)^{*}
$$

and therefore

$$
Q\left(a^{*}\right)=\overline{Q a} .
$$

The last formula follows from if we take ()$^{*}$ and ()$^{\prime}$ from the both side of the equation.

Lemma 5.6. Let $a, b$ be paravectors in $\mathcal{C} \ell_{0,3}$. Then $Q(a b)$ is a paravector. 
Proof. We just compute

$$
Q(a b)=Q a b^{\prime}+a Q b .
$$

Since $a, b$ are paravectors, the elements $Q a$ and $Q b$ are scalars, completing the proof.

Lemma 5.7. Let $\Omega \subset \mathbb{R}^{4}$ be open. A function $f: \Omega \rightarrow \mathcal{C} \ell_{0,3}$ is left $\alpha$ hypermonogenic if and only if $f^{*}$ is right $\alpha$-hypermonogenic.

Proof. Assume that $f$ is left $\alpha$-hypermonogenic then

$$
M_{\alpha}^{l} f(x)=x_{3} D^{l} f(x)+\alpha Q^{\prime} f(x)=0 .
$$

Since $\left(a^{*}\right)^{\prime}=\bar{a}$, we infer

$$
\begin{aligned}
0 & =\left(M_{\alpha}^{l} f(x)\right)^{*}=x_{3} D^{r} f^{*}+\alpha\left(Q^{\prime} f(x)\right)^{*} \\
& =x_{3} D^{r} f^{*}+\alpha(\bar{Q} f(x)) .
\end{aligned}
$$

Using the previous lemma we obtain

$$
M_{\alpha}^{r} f^{*}(x)=x_{3} D^{r} f^{*}+\alpha Q f^{*}(x)=0 .
$$

Hence $f^{*}$ is right $\alpha$-hypermonogenic. Similarly, we verify that if $f$ is right $\alpha$-hypermonogenic then $f$ is left $\alpha$-hypermonogenic.

Theorem 5.8. Let $\Omega$ be an open subset of $\mathbb{R}_{+}^{4}$ and $K \subset \Omega$ be a smoothly bounded compact set. Denote $\left(\nu_{0}, \nu_{1}, \nu_{2}, \nu_{3}\right)$ the outer unit normal and the corresponding paravector by $\nu=\nu_{0}+\nu_{1} e_{1}+\nu_{2} e_{2}+\nu_{3} e_{3}$. If $f$ is right $\alpha$ hypermonogenic in $\Omega$ and $a \in K$, then

$$
f(a)=\int_{\partial K}\left(P(f \nu) x_{3}^{-\alpha} h_{\alpha}(a, x)+Q(f \nu) e_{3} a_{3}^{\alpha} h_{-\alpha}(a, x)\right) d \sigma,
$$

where $h_{\alpha}(a, x)$ and $e_{3} a_{3}^{\alpha} h_{-\alpha}(a, x)$ are right $\alpha$-hypermonogenic with respect to the variable $a$.

Proof. If $f$ is right $\alpha$-hypermonogenic then $f^{*}$ is left $\alpha$-hypermonogenic and therefore

$$
f^{*}(a)=\int_{\partial K}\left(x_{3}^{-\alpha} h_{\alpha}(a, x) P\left(\nu f^{*}\right)+a_{3}^{\alpha} h_{-\alpha}(a, x) e_{3} Q^{\prime}\left(\nu f^{*}\right)\right) d \sigma .
$$

Taking ()$^{*}$ from the both sides we obtain

$$
f(a)=\int_{\partial K}\left(x_{3}^{-\alpha} P\left(\nu f^{*}\right)\right)^{*} h_{\alpha}^{*}(a, x)+\left(Q^{\prime}\left(\nu f^{*}\right)\right)^{*} e_{3} a_{3}^{\alpha} h_{-\alpha}^{*}(a, x) d \sigma .
$$

where $h_{\alpha}^{*}(a, x)=\left(h_{\alpha}(a, x)\right)^{*}$. Applying the previous lemma, we infer

$$
\left.\left(Q^{\prime}\left(\nu f^{*}\right)\right)^{*}=\overline{Q\left(\nu f^{*}\right.}\right)=Q(f \nu)
$$

and

$$
\left(P\left(\nu f^{*}\right)\right)^{*}=P(f \nu),
$$

since $f$ and $\nu$ are paravectors. Hence we have

$$
f(a)=\int_{\partial K}\left(x_{3}^{-\alpha} P(f \nu) h_{\alpha}^{*}(a, x)+(Q(f \nu)) e_{3} a_{3}^{\alpha} h_{-\alpha}^{*}(a, x)\right) d \sigma .
$$


Since $h_{\alpha}^{a}$ is a paravector we infer

$$
f(a)=\int_{\partial K}\left(x_{3}^{-\alpha} P(f \nu)\left(h_{\alpha}\right)(a, x)+(Q(f \nu)) e_{3} a_{3}^{\alpha}\left(h_{-\alpha}\right)(a, x)\right) d \sigma
$$

completing the proof.

Theorem 5.9. Let $\Omega$ be an open subset of $\mathbb{R}_{+}^{4}$ and $K \subset \Omega$ be a smoothly bounded compact set. Denote $\left(\nu_{0}, \nu_{1}, \nu_{2}, \nu_{3}\right)$ the outer unit normal and the corresponding paravector by $\nu=\nu_{0}+\nu_{1} e_{1}+\nu_{2} e_{2}+\nu_{3} e_{3}$. Then, if $f$ is a paravector valued $\alpha$-hypermonogenic in $\Omega$ and $a \in K$,

$$
\begin{aligned}
f(a)= & \int_{\partial K}\left(h_{\alpha}(a, x)\langle\bar{\nu}, f\rangle+\left[P h_{\alpha}(a, x), \overline{P \nu}, P f\right]\right) \frac{d \sigma}{x_{3}^{\alpha}} \\
& -\int_{\partial K} a_{3}^{a}\left(\left[h_{-\alpha}(a, x), \overline{P \nu}, Q f e_{3}\right]+\left[h_{-\alpha}(a, x), Q \nu e_{3}, P f\right]\right) d \sigma .
\end{aligned}
$$

Proof. If $f$ is a paravector valued $\alpha$-hypermonogenic in $\Omega$ and $a \in K$, then

$$
\begin{aligned}
f(a) & =\frac{1}{2} \int_{\partial K}\left(x_{3}^{-\alpha} P(f \nu) h_{\alpha}(a, x)+Q(f \nu) e_{3} a_{3}^{\alpha} h_{-\alpha}(a, x)\right) d \sigma \\
& +\frac{1}{2} \int_{\partial K}\left(x_{3}^{-\alpha} h_{\alpha}(a, x) P(\nu f)+h_{-\alpha}(a, x) e_{3} a_{3}^{\alpha} Q^{\prime}(\nu f)\right) d \sigma
\end{aligned}
$$

Since $P(\nu f)=P \nu P f+Q \nu Q^{\prime} f$ and $f$ is a paravector we obtain

$$
\begin{aligned}
\frac{1}{2} \int_{\partial K} x_{3}^{-\alpha} h_{\alpha}(a, x) P(\nu f)+x_{3}^{-\alpha} P(f \nu) h_{\alpha}(a, x) d \sigma \\
=\frac{1}{2}\left(\int_{\partial K} x_{3}^{-\alpha} h_{\alpha}(a, x) P \nu P f+x_{3}^{-\alpha} P f P \nu h_{\alpha}(a, x)\right) d \sigma \\
\quad-\int_{\partial K} x_{3}^{-\alpha} h_{\alpha}(a, x) Q \nu Q f d \sigma \\
=\int_{\partial K} x_{3}^{-\alpha} h_{\alpha}(a, x)\langle\bar{\nu}, f\rangle d \sigma-\int_{\partial K} x_{3}^{-\alpha}\left[h_{\alpha}(a, x), \overline{P \nu}, P f\right] d \sigma \\
=\int_{\partial K} x_{3}^{-\alpha} h_{\alpha}(a, x)\langle\bar{\nu}, f\rangle d \sigma+\int_{\partial K} x_{3}^{-\alpha}\left[h_{\alpha}(a, x), \overline{P \nu}, P f\right] d \sigma .
\end{aligned}
$$

Similarly we compute

$$
\begin{aligned}
\frac{1}{2} \int_{\partial K} h_{-\alpha}(a, x) e_{3} a_{3}^{\alpha} Q^{\prime}(\nu f)+Q(f \nu) e_{3} a_{3}^{\alpha}\left(h_{-\alpha}\right)(a, x) d \sigma \\
=\frac{1}{2} \int_{\partial K} h_{-\alpha}(a, x) a_{3}^{\alpha} P \nu Q f e_{3}+Q f a_{3}^{\alpha} e_{3} P \nu a_{3}^{\alpha}\left(h_{-\alpha}\right)(a, x) d \sigma \\
\quad+\frac{1}{2} \int_{\partial K} h_{-\alpha}(a, x) a_{3}^{\alpha} Q \nu e_{3} P f+P f a_{3}^{\alpha} Q \nu e_{3}\left(h_{-\alpha}\right)(a, x) d \sigma \\
=-\int_{\partial K} a_{3}^{a}\left[h_{-\alpha}(a, x), \overline{P \nu}, Q f e_{3}\right]-\int_{\partial K} a_{3}^{\alpha}\left[h_{-\alpha}^{a}(a, x), Q \nu e_{3}, P f\right] d \sigma,
\end{aligned}
$$

completing the proof. 


\section{Acknowledgements}

Open access funding provided by University of Helsinki including Helsinki University Central Hospital.

Open Access. This article is distributed under the terms of the Creative Commons Attribution 4.0 International License (http://creativecommons.org/licenses/by/4. $0 /$ ), which permits unrestricted use, distribution, and reproduction in any medium, provided you give appropriate credit to the original author(s) and the source, provide a link to the Creative Commons license, and indicate if changes were made.

Publisher's Note Springer Nature remains neutral with regard to jurisdictional claims in published maps and institutional affiliations.

\section{References}

[1] Akin, Ö., Leutwiler, H.: On the invariance of the solutions of the Weinstein equation under Möbius transformations, Classical and modern hypercomplextential theory and applications (Chateau de Bonas, 1993), pp. 19-29, NATO Adv. Sci. Inst. Ser. C Math. Phys. Sci., vol. 430. Kluwer, Dordrecht (1994)

[2] Eriksson, S.-L.: Integral formulas for hypermonogenic functions. Bull. Belgian Math. Soc. 11, 705-717 (2004)

[3] Eriksson, S.-L.: Hyperbolic extensions of integral formulas. Adv. Appl. Clifford Algebras 20(3-4), 575-586 (2010)

[4] Eriksson, S.-L., Orelma, H.: General integral formulas for $k$-hypermonogenic functions. Adv. Appl. Clifford Algebras 27(1), 99-110 (2017)

[5] Eriksson, S.-L., Orelma, H.: A new Cauchy type integral formula for quaternionic $k$-hypermonogenic functions. In: Bernstein, S., Kahler, U., Sabadini, I., Sommen, F. (eds.) Modern Trends in Hypercomplex Analysis, Trends in Mathematics, pp. 175-189. Springer International Publishing, Cham (2016)

[6] Eriksson, S.-L., Orelma, H.: Quaternionic hyperbolic function theory. In: Topics in Clifford Analysis A Special Volume in Honor of Wolfgang Sprößig, Editors Swanhild Bernstein in the Springer series Trends in Mathematics (TIM) (2019) (to appear)

[7] Eriksson, S.-L., Vuojamo, V.: Integral kernels for k-hypermonogenic functions. Complex Var. Elliptic Equ. 62(9), 1254-1265 (2017). https://doi.org/10.1080/ 17476933.2016.1250402

[8] Eriksson-Bique, S.-L.: $k$-hypermonogenic functions. In: Begerh, H., Gilbert, R., Wong, M.W. (eds.) Progress in Analysis, pp. 337-348. World Scientific, Singapore (2003)

[9] Eriksson-Bique, S.-L., Leutwiler, H.: Hypermonogenic functions. In: Clifford Algebras and their Applications in Mathematical Physics, vol. 2. Birkhäuser, Boston, pp. 287-302.x (2000)

[10] Gürlebeck, K., Habetha, K., Sprößig, W.: Holomorphic Functions in the Plane and $n$-dimensional Space. Birkhäuser, Basel (2008)

[11] Hempfling, Th., Leutwiler, H.: Modified Quaternionic Analysis in $\mathbb{R}^{4}$. In: Clifford Algebras and Their Application in Mathematical Physics, Fundamental Theories of Physics, vol. 94, pp. 227-237 
[12] Leutwiler, H.: Appendix: Lecture notes of the course "Hyperbolic harmonic functions and their function theory". Clifford algebras and potential theory, pp. 85-109, Univ. Joensuu Dept. Math. Rep. Ser., vol. 7. Univ. Joensuu, Joensuu (2004)

[13] Orelma, H.: New Perspectives in Hyperbolic Function Theory. TUT, Dissertation, 892 (2010)

Sirkka-Liisa Eriksson

Department of Mathematics and Statistics

University of Helsinki

P.O. Box 68

00014 University of Helsinki

Finland

e-mail: Sirkka-Liisa.Eriksson@helsinki.fi

Heikki Orelma

Laboratory of Civil Engineering

Tampere University

Tampere

Finland

e-mail: Heikki.Orelma@tuni.fi

Received: November 21, 2018.

Accepted: September 23, 2019. 